\title{
Análisis de la población de condrictios (Vertebrata: Chondrichthyes) de aguas demersales y profundas del Caribe centroamericano, a partir de faenas de prospección pesquera con redes de arrastre
}

\author{
Population analysis of demersal and deep water \\ chondrichthyes (Vertebrata: Chondrichthyes) along the \\ Caribbean coast of Central America based on a fishery survey \\ using bottom trawling
}

\author{
Rosario Benavides M..$^{{ }^{*}}$, Carlos Luis Brenes $R .{ }^{1}$ y Amaru Márquez A. ${ }^{1}$
}

\begin{abstract}
RESUMEN
Se describen la abundancia, riqueza, diversidad y distribución de las especies de condrictios de profundidad, capturadas en campaña de prospección pesquera del B/O Miguel Oliver en la plataforma y el talud continental de la costa Caribe de Centroamérica, entre el 3 de enero del 2011 y el 3 de febrero del 2011. Se utilizó una red tipo Lofoten para realizar arrastres de fondo de 30 minutos de duración cada uno, en 96 puntos de muestreo de los 25 transectos equidistantes. Se capturaron 28 especies, pertenecientes a 6 órdenes y 11 familias. Se describen 8 especies de rayas, 5 de quimeras y 15 de tiburones. La familia Squalidae mostró la mayor abundancia $(\mathrm{n}=184)$, mayor riqueza $(7$ especies $)$ y biomasa $(222.6 \mathrm{~kg}$, $58 \%$ ), donde la especie dominante fue $S$. cubensis representada por un $37 \%$ en las capturas. El valor más alto del índice de diversidad de Shannon se obtuvo frente a las costas de Nicaragua (2.051). La distribución de las especies por temperatura y profundidad las ubica entre los 4 y $16^{\circ} \mathrm{C}$ y entre los 20 y $1000 \mathrm{~m}$, respectivamente. Se reporta por primera vez en esta área la presencia de ocho especies: $H$. mirabilis, $R$. atlantica, D. profundorum, C. tesselatus, E. gracilispinis, E. carteri, G. springeri y H. perlo.
\end{abstract}

Palabras claves: Condrictios, Caribe, Centroamérica, plataforma continental, arrastre.

\begin{abstract}
The abundance, richness, diversity, and distribution are described for deep water chondrichthyan fauna collected during a survey of Oceanographic Vessel Miguel Oliver along the continental shelf on the Caribbean coast of Central America from January $3^{\text {rd }}$ to February $3^{\text {rd }}$, 2011. A Lofoten net was used for bottom trawling for 30 minutes, in each of 96 different sampling points distributed along 25 equidistant transects. A total of 28 species was collected belonging to six orders and ten families. In addition, 8 species of rays, 5 species of chimaeras and 15 species of sharks are described. The Squalidae family showed the highest abundance $(n=184)$, richness (7 species) and biomass (222.6kg, 58\%). The dominant species was $S$. cubensis represented by $37 \%$ collected. The highest Shannon Diversity Index was obtained along the Nicaraguan coasts
\end{abstract}

Laboratorio de Oceanografía y Manejo Costero, Universidad Nacional, Heredia, Costa Rica, Apdo. 86-3000; rbmorera@gmail.com*,cbrenes.una@gmail.com,tupac.marquez@gmail.com

Recibido: 5 de octubre de 2013

Corregido: 23 de noviembre de 2013

Aceptado: 28 de febrero de 2014

DOI: http://dx.doi.org/10.15359/revmar.6.1 
(2.051). The distribution of species by temperature and depth was between 4 and $16^{\circ} \mathrm{C}$ and between 20 and $1,000 \mathrm{~m}$. In addition, eight species were reported for the first time for this area $H$. mirabilis, $R$. atlantica, D. profundorum, C. tesselatus, E. gracilispinis, E. carteri, G. springeri and $H$. perlo.

Keywords: Chondrichtyes, Caribbean, Central America, continental shelf, trawling.

\section{INTRODUCCIÓN}

El mar Caribe es una cuenca semicerrada que pertenece al océano Atlántico occidental, bordeado por las costas de Centro y Suramérica y por las Antillas. Comprende un área de $2754000 \mathrm{~km}^{2}$, un volumen de aproximadamente $6.5 \times 10^{6} \mathrm{~km}^{3} \mathrm{y}$ más de $13500 \mathrm{~km}$ de línea de costa (Miloslavich et al. 2010).

La región costera caribeña es un gran ecosistema marino que se caracteriza por la presencia de arrecifes de coral, manglares y praderas marinas, incluyendo también otros ambientes como playas arenosas y rocosas. Este ecosistema tropical incorpora una gran diversidad de flora y fauna asociada, y los países que bordean esa zona abarcan colectivamente una gran biodiversidad marina global (Miloslavich et al. 2010). El Caribe centroamericano es considerado una región biogeográficamente única que posee una compleja historia geológica (Alvarado et al. 2008). En esa zona, la plataforma continental es de 125000 $\mathrm{km}^{2}$ de extensión y tiene cerca de 3 $800 \mathrm{~km}$ de línea de costa (OSPESCA, 2009). La plataforma continental de Belice, Costa Rica y Panamá es angosta, mientras que en Honduras y Nicaragua es amplia.

Según Miloslavich \& Klein (2005), esaregión tiene ecosistemas de plataforma con cuencas profundas y trincheras parcialmente aisladas, las cuales son habitadas por una gran cantidad de especies. Sin embargo, el conocimiento científico sobre la biodiversidad en la región es limitado, siendo una de las zonas menos conocidas en cuanto a la descripción taxonómica de su biota (Alvarado et al. 2008), como sucede con las poblaciones de condrictios. La mayoría de trabajos publicados se enfocan en especies del Atlántico noroccidental, del Golfo de México y del Caribe sur, y no así en el Caribe centroamericano (Hernández \& Núñez, 1998; Konstantinou et al. 2000; Baum et al. 2003; Large et al. 2003; Acevedo et al. 2007; Caldas et al. 2009a; Tavares, 2009).

Taxonómicamente, la clase condrictios incluye dos subclases llamadas Elasmobranchii y Holocephali; en la primera, se ubican los tiburones y rayas, mientras que en la segunda, las quimeras (Nelson, 2006). Los peces cartilaginosos son especies importantes por su interés ecológico y por su valor económico, y los tiburones son uno de los grupos más explotados y, por ende, sensibles a la actividad pesquera (Tavares, 2009). Se conocen como organismos pertenecientes a la estrategia de vida $K$, lo que significa que son individuos de crecimiento lento, con madurez sexual tardía y baja fecundidad. Por esta razón, difícilmente soportan un ritmo acelera- 
do de explotación. La mayoría de los tiburones son depredadores tope, se ubican en el ápice de la cadena trófica y desempeñan una función esencial en el mantenimiento del equilibrio de los ecosistemas marinos, principalmente en las regiones tropicales (Bascompte et al. 2005). Los batoideos comprenden más especies que todos los otros nueve órdenes de la clase combinados. El número total de especies de rayas representa más del $22 \%$ de los condrictios conocidos, y cerca del $43 \%$ de todos los batoideos. Esos organismos son primordialmente bentónicos marinos que habitan aguas desde la línea de marea baja hasta los $3000 \mathrm{~m}$ de profundidad, prefiriendo hábitats de fondo con sustratos suaves y son cosmopolitas (Ebert \& Compagno, 2007).

Las quimeras constituyen un particular linaje de peces que en la actualidad habitan principalmente las aguas profundas de todos los océanos. Se conocen entre 31 y 50 especies en este pequeño grupo (González et al. 2007).

Los condrictios se distribuyen a lo largo de todos los océanos del mundo. Compagno et al. (1989) señalan que el $55 \%$ de ellos se pueden encontrar a profundidades de hasta $200 \mathrm{~m}$, el $35 \%$ en la plataforma continental, el $2 \%$ en la zona oceánica y el $8 \%$ restante en otros ambientes. Dada la diversidad y distribución de las especies de ese grupo alrededor del mundo, esas poblaciones son aprovechadas por pesquerías industriales, artesanales, tradicionales y deportivas. Los sistemas de pesca utilizados para sus capturas son variados, al igual que el uso de los ejemplares y/o sus subproductos.
A pesar de su conocida vulnerabilidad a la sobrepesca, los tiburones han sido explotados cada vez más en décadas recientes, tanto por la pesca de descarte de las flotas de línea larga pelágicas como por las pesquerías directas (Baum et al. 2003).

Los tiburones y las rayas son miembros recurrentes de la llamada FACA (fauna de acompañamiento), producto de las pesquerías de arrastre, no obstante, muchas especies de condrictios de profundidad especialmente del Caribe centroamericano tienen descripciones deficientes o carecen de análisis exhaustivos de sus poblaciones (Mejía-Falla et al. 2007; Kyne et al. 2012), debido probablemente a que no existe una flota pesquera de arrastre de profundidad en esta región del Caribe. En este contexto, el objetivo primordial del presente trabajo es brindar información biológica importante sobre este grupo de peces que frecuentan la plataforma y el talud continental del Caribe centroamericano.

\section{MATERIALES Y MÉTODOS}

Área de estudio: La prospección pesquera fue realizada del 3 de enero del 2011 al 3 de febrero del 2011, abarcó la plataforma y el talud continental del Caribe centroamericano, desde el sur de Panamá hasta el norte de Belice (Fig. 1).

Arte de pesca y equipamientos: La faena de pesca se realizó a bordo del Buque Oceanográfico (B/O) Miguel Oliver, el cual tenía una red de arrastre de fondo tipo Lofoten (arrastre con puertas), de 2 tapas de paño polietileno para fondos duros, cuya longitud total era de $48.7 \mathrm{~m}$; copo de 14.3 


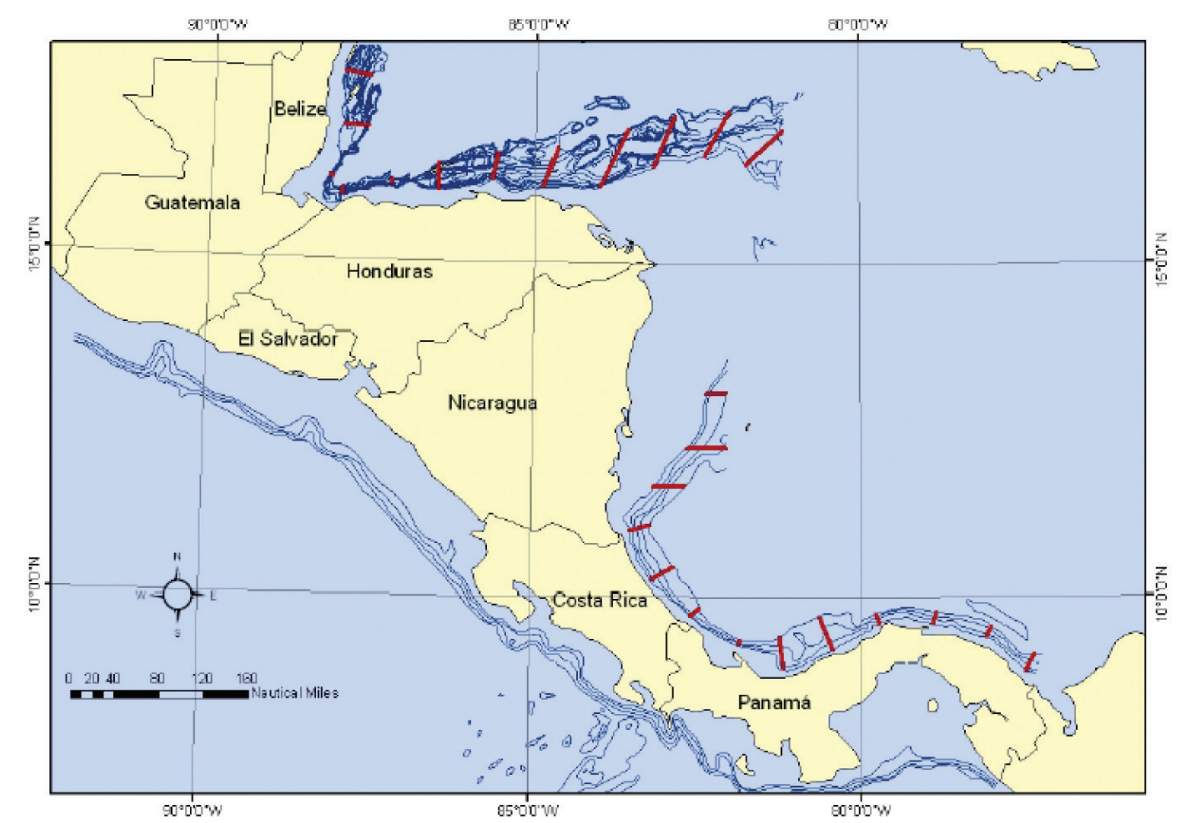

Fig. 1. Área de estudio y transectos equidistantes y perpendiculares a la línea de costa Fig. 1. Study area and equidistant transects perpendicular to the coastline

$\mathrm{m}$ de largo y $35 \mathrm{~mm}$ de luz de malla de Poliamida y tren de arrastre de $41.7 \mathrm{~m}$ de longitud de $1000 \mathrm{~kg}$ de peso aproximado.

Metodología de pesca: Se realizaron 96 lances con una duración de $30 \mathrm{~min}$. de arrastre efectivo, contando desde el momento del firme del lance en el fondo hasta el inicio de la orden de virada. El control del tiempo de arrastre se realizó mediante el empleo de los sensores de red (ITI-SCANMAR). Las faenas de pesca fueron diurnas.

Diseño de muestreo: El estudio se desarrolló en la plataforma y el talud continental hasta los $1500 \mathrm{~m}$ de profundidad. El muestreo se llevó a cabo con arrastre de fondo en puntos previamente establecidos en transectos perpendiculares a la costa. Para cubrir el área de estudio, se realizaron un total de 25 transectos equidistantes (Fig. 1). Cuando las características del fondo fueron favorables para el arrastre, se efectuaron 5 o 6 lances en cada uno de ellos y, adicionalmente, se tomaron datos fisicoquímicos en cada lance.

En cada punto de muestreo se registraron la captura total de condrictios, el número de ejemplares y su biomasa. El número de ejemplares se determinó por conteo directo en el buque, donde los especímenes eran mantenidos hasta que finalizara el trabajo de virado de la red en cada punto, para ser devueltos posteriormente al mar. Durante los muestreos biológicos se registraron la longitud total $(\mathrm{cm})$ por medición directa con un ictiómetro, el peso y sexo de cada uno de los especímenes.

Análisis de datos: Se determinó la diversidad del ensamblaje de condrictios a través del índice de Shannon-Wiener (Peet, 1975; Magurran, 1988; Moreno, 
2001). Se empleó el índice de equidad de Pielou para medir la proporción de la diversidad observada en cada estación en relación con la máxima diversidad esperada. El coeficiente de similitud de Jaccard fue utilizado para expresar el grado en el que dos muestras son semejantes por las especies presentes en ellas, siendo una medida inversa de la diversidad, que se refiere al cambio de especies entre dos estaciones (Pielou, 1975; Magurran, 1988). Finalmente, para evaluar la composición de las comunidades encontradas se utilizó un escalamiento multidimensional no métrico (NMDS) de los valores de abundancias estandarizados para cada lance y se ajustaron los valores de temperatura y profundidad para visualizar las relaciones existentes (Borcard et al. 2011).

\section{RESULTADOS}

En el Cuadro 1 se muestran los resultados de las 28 especies encontradas durante las faenas de pesca, 8 especies pertenecen a los batoideos, 5 correspondieron a Holocéfalos, y finalmente, 15 correspondieron a tiburones.

La abundancia de especímenes por familia estuvo dominada por Squalidae, con 184 ejemplares, lo que representa el $49 \%$ de la captura, seguida por la familia Scyliorhinidae, con 65 ejemplares (17\%). La familia Rhinochimaeridae ocupó el tercer lugar en abundancia, con 63 ejemplares (16.5\%). Las otras familias son poco abundantes en el área (Fig. 2A).

Por su parte, Squalus cubensis resultó ser la especie más abundante con un $37 \%$, seguida de Neoharriotta carri $(16 \%)$ y Galeus arae (15\%) (Fig. 2B).
La abundancia total de especímenes capturada por país presentó valores máximos en Panamá (90), seguida de Honduras (60), y valores mínimos en Costa Rica (18) (Fig. 3). En términos de riqueza, el valor máximo se observó en Honduras (17) y el valor mínimo se obtuvo en Costa Rica (2) (Fig. 4).

La familia con mayor riqueza fue Squalidae (siete), lo que representa una cuarta parte del total, seguida por Rajidae y Scyliorhinidae (cinco). Rhinochimaeridae (tres); Chimaeridae y Dasyatidae (dos). Anacanthobatidae, Triakidae, Squatinidae y Hexanchidae, cada una representada por una especie (Cuadro 1).

La captura total en biomasa de condrictios fue de $384 \mathrm{~kg}$, la mayor biomasa la obtuvo la familia Squalidae, con 222.6 kg (58\%), seguida por Dasyatidae, con $66.5 \mathrm{~kg}(17 \%)$. Las restantes familias presentaron valores bajos a la biomasa total (Fig. 5, Cuadro 2).

La diversidad fue mayor en Nicaragua $\left(H^{\prime}=2.051\right)$, mientras que Costa Rica presentó los menores valores $\left(\mathrm{H}^{\prime}=\right.$ 0.562). La equidad presentó valores similares a los de diversidad, con un valor máximo de 0.891 en Nicaragua y un mínimo de 0.623 en Panamá (Cuadro 3).

Los resultados del presente estudio muestran en el índice de similitud de Jaccard que todos los países centroamericanos comparten al menos una especie de condrictios, a excepción de Costa Rica y Nicaragua, mientras que Panamá y Honduras tuvieron más similitud en composición de especies (Cuadro 4).

Del análisis de los rangos de temperatura y profundidad de las capturas, se ob- 
Cuadro 1. Orden, familia, especie e informe previo para el Caribe centroamericano de las especies de condrictios encontradas en el muestreo realizado Table 1. Order, family, species and preliminary report of chondrichthyes sampled in the Caribbean waters of Central America

\begin{tabular}{|c|c|c|c|}
\hline Familia & Especie & Autoridad & \\
\hline \multirow[t]{8}{*}{ Rajiformes } & Anacanthobatidae & $\begin{array}{l}\text { Anacanthobatis } \\
\text { americanus }\end{array}$ & Bigelow \& Schroeder, 1962 \\
\hline & Dasyatidae & Dasyatis americana & Hildebrand \& Schroeder, 1928 \\
\hline & & Dasyatis guttata & (Bloch \& Schneider, 1801) \\
\hline & Rajidae & Breviraja nigriventralis & McEachran \& Matheson, 1985 \\
\hline & & Gurgesiella atlantica & (Bigelow \& Schroeder, 1962) \\
\hline & & Dipturus bullisi & (Bigelow \& Schroeder, 1962) \\
\hline & & Cruriraja rugosa & Bigelow \& Schroeder, 1958 \\
\hline & & Leucoraja yucatanensis & (Bigelow \& Schroeder, 1950) \\
\hline \multirow[t]{5}{*}{ Chimaeriformes } & Chimaeridae & Hydrolagus mirabilis* & (Collett, 1904) \\
\hline & & Hydrolagus alberti & Bigelow \& Schroeder, 1951 \\
\hline & & Chimaera cubana & Howell Rivero, 1936 \\
\hline & Rhinochimaeridae & $\begin{array}{l}\text { Rhinochimaera } \\
\text { atlantica* }\end{array}$ & Holt \& Byrne, 1909 \\
\hline & & Neoharriotta carri & Bullis \& Carpenter, 1966 \\
\hline \multirow[t]{7}{*}{ Squaliformes } & Squalidae & Deania profundorum* & (Smith \& Radcliffe, 1912) \\
\hline & & Squalus cubensis & Howell Rivero, 1936 \\
\hline & & $\begin{array}{l}\text { Centrophorus } \\
\text { granulosus }\end{array}$ & (Bloch \& Schneider, 1801) \\
\hline & & $\begin{array}{l}\text { Centrophorus } \\
\text { tesselatus* }\end{array}$ & Garman, 1906 \\
\hline & & $\begin{array}{l}\text { Etmopterus } \\
\text { gracilispinis* }\end{array}$ & Krefft, 1968 \\
\hline & & Etmopterus carteri ${ }^{*}$ & Springer \& Burgess, 1985 \\
\hline & & Etmopterus schultzi & $\begin{array}{c}\text { Bigelow, Schroeder \& Springer, } \\
1953\end{array}$ \\
\hline \multirow[t]{6}{*}{ Carchariniformes } & Scyliorhinidae & Galeus arae & (Nichols, 1927) \\
\hline & & Galeus springeri* & Konstantinou \& Cozzi, 1998 \\
\hline & & Apristurus parvipinnis & Springer \& Heemstra, 1979 \\
\hline & & Apristurus riveri & Bigelow \& Schroeder, 1944 \\
\hline & & $\begin{array}{l}\text { Schroderichthys } \\
\text { maculatus }\end{array}$ & Springer, 1966 \\
\hline & Triakidae & Mustelus norrisi & Springer, 1939 \\
\hline Squatiniformes & Squatinidae & Squatina dumeril & Lesueur, 1818 \\
\hline Hexanchiformes & Hexanchidae & Heptranchias perlo* & (Bonnaterre, 1788) \\
\hline
\end{tabular}

\section{* Primeros registros para el Caribe centroamericano}



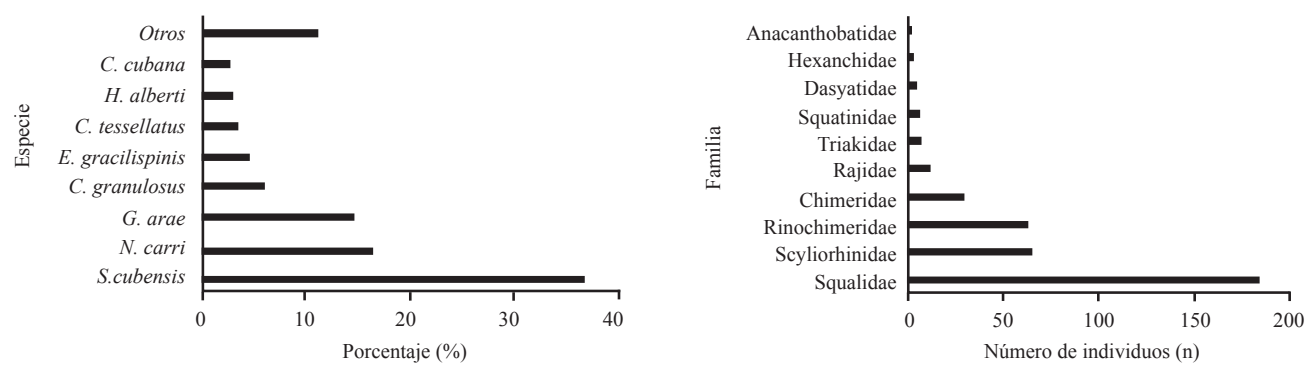

Fig. 2. Abundancia de especímenes por familias (A) y por especie (B) de los condrictios capturados en el Caribe centroamericano

Fig. 2. Abundance of specimens by family (A) and species (B) of chondrichthyans caught in the Caribbean waters of Central America

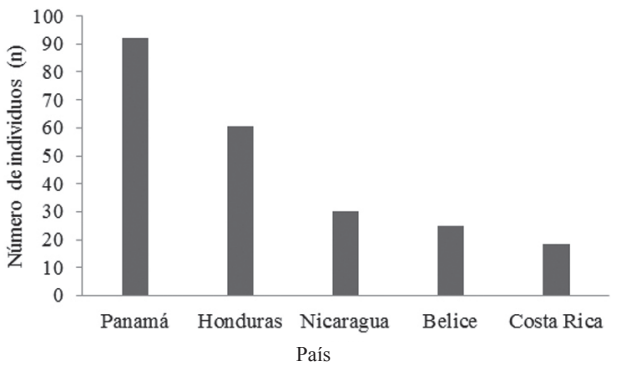

Fig. 3. Abundancia de especímenes de condrictios capturados por país en el Caribe centroamericano

Fig. 3. Abundance of chondrichthyes specimens captured by country in the Caribbean waters of Central America

tuvo que la mayoría de las especies (22) se concentraron en temperaturas entre los 4 y $16^{\circ} \mathrm{C}$, y las profundidades a las que se les capturó oscilaron entre los 20 y 1000 $\mathrm{m}$. Las seis especies restantes se encuentran fuera de los rangos descritos: Dasyatis guttata, Etmopterus carteri, Mustelus norrisi, Deania profundorum, Apristurus riveri y Rhinochimaera atlantica (Fig. 6).

Muchas delas especies señaladas ya habían sido registradas anteriormente en el área o en las periferias, sin embargo, algunas especies tales como, Hydrolagus mirabilis, $R$. atlantica, D.

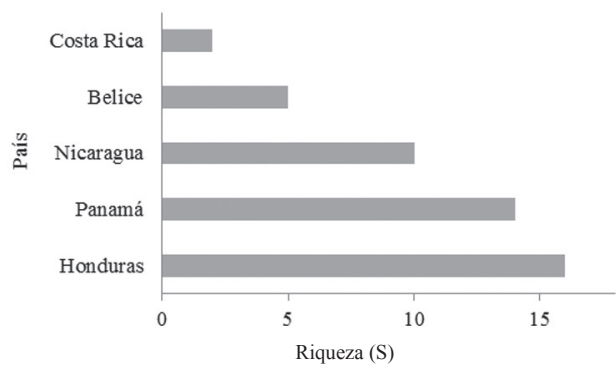

Fig. 4. Riqueza de condrictios capturados en el Caribe centroamericano

Fig. 4. Specific richness of chondrichthyes collected along the Caribbean waters of Central America

profundorum, Centrophorus tesselatus, Etmopterus gracilispinis, E. carteri, Galeus springeri y Heptranchias perlo no tenían reportes previos en la zona ni en las profundidades específicas del estudio (Cuadro 1, Fig. 7).

Aunque la distribución de las especies no es homogénea, $S$. cubensis y E. gracilispinis fueron capturadas en las costas de todos los países centroamericanos excepto en Costa Rica, con la diferencia de que la primera había sido reportada para estas áreas, mientras que la segunda no lo había sido 


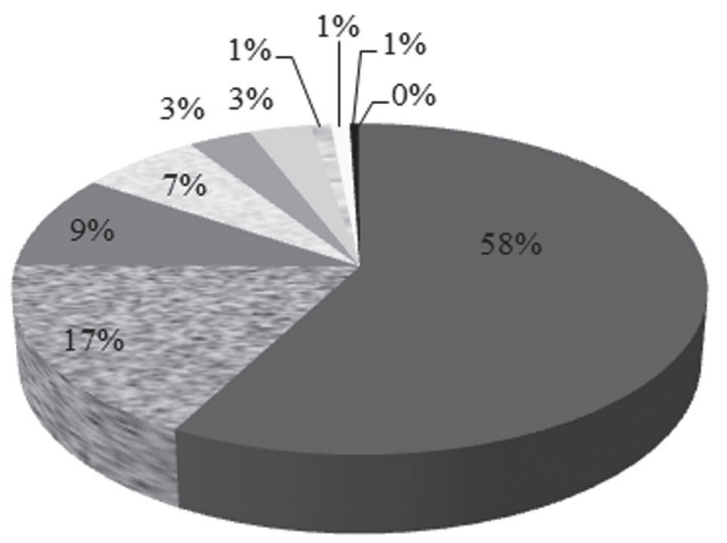

- Squalidae
@ Dasyatidae
- Rhinochimaeridae
Chimaeridae
Triakidae
Squatinidae
Scyliorhinidae
Hexanchidae
- Rajidae
Anacanthobatidae

Fig. 5. Contribución en porcentaje de cada familia de condrictios a la biomasa total capturada en enero del 2011

Fig. 5. Contribution percentage of each chondrichthyan family to total biomass collected in January 2011

nunca (Cuadro 1). H. mirabilis, especie que tampoco había sido reportada en el Caribe centroamericano, fue capturada en las costas de Honduras, Costa Rica y Panamá. $R$. atlantica, $C$. tesselatus y $H$. perlo tampoco habían sido reportadas en el Caribe centroamericano, y fueron capturadas solamente en Panamá. D. profundorum, G. springeri y E. carteri fueron capturadas en las costas de Honduras (Fig. 7). La mayor amplitud de distribución batimétrica fue observada en Chimaera cubana, la cual se le capturó entre los 270-1 $050 \mathrm{~m}$ de profundidad (Cuadro 2).

A continuación se presenta una breve descripción de las especies reportadas como nuevos registros para la zona.

Hydrolagus mirabilis (Collett, 1904)

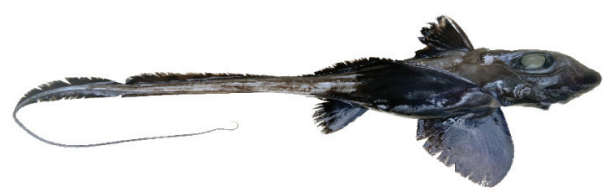

Se distribuye en aguas del Atlántico este y norte, principalmente. Vive a profundidades que van desde los 450 hasta los 1933 m (Kyne \& Simpfendorfer, 2007). En el presente trabajo se capturó en Panamá, Costa Rica y Honduras, entre los 500 y $1250 \mathrm{~m}$. Presenta un cuerpo con una cabeza prominente y termina en una larga cola en forma de filamento, además, un hocico corto y cónico, y una piel lisa, excepto en los órganos sexuales, donde tiene dentículos. Su color es café oscuro uniforme y puede llegar a medir $80 \mathrm{~cm}$ LT (el tamaño de su filamento sin dañar comprende un tercio de este). Tiene hábitos bentopelágicos sobre el talud continental.

Rhinochimaera atlantica Holt \& Byrne, 1909

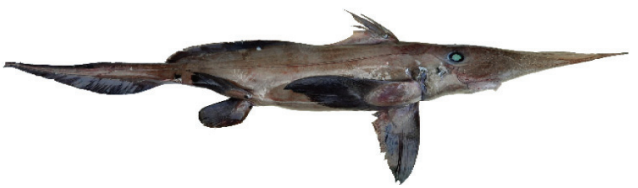


Cuadro 2. Caracteres descriptivos de los ejemplares capturados: Sexo = M-Macho y HHembra (entre paréntesis se indica el número de especímenes por sexo); LT $(\mathrm{cm})=$ Longitud Total (entre paréntesis se indican el valor mínimo y el valor máximo); Biomasa $(\mathrm{kg})$ = peso total por sexo; País = área donde se capturaron los condrictios (Pan-Panamá, Hon-Honduras, Nic-Nicaragua, CR-Costa Rica; Bel-Belice); y Profundidad (m) = rango de profundidad de las estaciones en las cuales los ejemplares fueron capturados Table 2. Descriptive features of specimens caught: Sex = M-Male and H-Female (number of specimens by sex indicated in parenthesis); LT $(\mathrm{cm})=$ total length (minimum and maximum values in parenthesis); Biomass $(\mathrm{kg})=$ total weight by species and sex; Country $=$ area where chondrichthyes were collected (Pan-Panama, Hon-Honduras, Nic-Nicaragua, CR-Costa Rica; Bel-Belize); and Depth $(\mathrm{m})=$ depth range of the stations where specimens were collected

\begin{tabular}{|c|c|c|c|c|c|}
\hline Especie & Sexo & LT (cm) & Biomasa (kg) & País & Profundidad \\
\hline A. americanus & $M:(n=2)$ & $16-17$ & 146 & Pan. & $550-700$ \\
\hline D. americana & $M:(n=1) ; H:(n=1)$ & $48 ; 49$ & $3000 ; 3170$ & Hon. & $240-280$ \\
\hline D. guttata & $\mathrm{H}:(\mathrm{n}=3)$ & $62-202$ & 60429 & Nic. & $200-230$ \\
\hline B. nigriventralis & $\mathrm{H}:(\mathrm{n}=1)$ & 13 & 68 & Pan. & 550 \\
\hline G. atlantica & $\mathrm{H}:(\mathrm{n}=2)$ & $13-15$ & 60 & Nic. & $400-450$ \\
\hline D. bullisi & $M:(n=1)$ & 21 & 118 & Nic. & $700-830$ \\
\hline C. rugosa & $M:(n=2) ; H:(n=1)$ & $20 ; 23$ & $106 ; 322$ & Hon. & $450-550$ \\
\hline L. yucatanensis & $M:(n=2) ; H:(n=3)$ & $18 ; 13-21$ & $308 ; 904$ & Hon. & $250-500$ \\
\hline H. mirabilis & $M:(n=2) ; H:(n=5)$ & $36-57.5 ; 16-57$ & $1103 ; 2052$ & Pan.; CR.; Hon. & $500-1250$ \\
\hline H. alberti & $M:(n=6) ; H:(n=6)$ & $45.5-55 ; 22-60.5$ & $4953 ; 4302$ & Pan.; CR.; Hon.; Bel. & $590-770$ \\
\hline N. carri & $M:(n=31) ; H:(n=31)$ & $28.5-54.5 ; 28.5-54.5$ & $14375 ; 18609$ & Pan.; Hon. & $350-550$ \\
\hline R. atlantica & $\mathrm{H}:(\mathrm{n}=1)$ & 79 & 2692 & Pan. & $1450-1500$ \\
\hline C. cubana & $M:(n=7) ; H:(n=3)$ & $46-59 ; 21-62.5$ & $8578 ; 3728$ & Nic.; Hon. & $270-1050$ \\
\hline D. profundorum & I & 70 & 1294 & Hon. & $750-800$ \\
\hline S. cubensis & $M:(n=65) ; H:(n=75)$ & $33-57 ; 24-100$ & $21198 ; 45631$ & Pan.; Nic.; Hon.; Bel. & $150-900$ \\
\hline C. granulosus & $M:(n=11) ; H:(n=12)$ & $85-106 ; 44-160$ & $49786 ; 71712$ & Hon.; Nic.; Pan. & $350-900$ \\
\hline C. tesselatus & $M:(n=1) ; H:(n=3)$ & $76 ; 93-96$ & $1840 ; 11766$ & Pan. & $750-950$ \\
\hline E. gracilispinis & $M:(n=6) ; H:(n=11)$ & $26-53 ; 26-106$ & $2925 ; 16299$ & Nic.; Bel.; Hon.; Pan. & $750-1150$ \\
\hline E. carteri & $\mathrm{H}:(\mathrm{n}=1)$ & 21 & 38 & Hon. & $450-500$ \\
\hline E. schultzi & $M:(n=1)$ & 29 & 96 & Hon. & $700-750$ \\
\hline G. arae & $M:(n=46) ; H:(n=10)$ & $23-32 ; 22-30$ & $2679 ; 442$ & Pan.; Hon.; Bel. & $400-550$ \\
\hline G. springeri & $\mathrm{H}:(\mathrm{n}=1)$ & 52 & 432 & Bel. & $500-550$ \\
\hline A. parvipinnis & $M:(n=1)$ & 14 & 180 & Nic. & $800-850$ \\
\hline A. riveri & $\mathrm{H}:(\mathrm{n}=1)$ & 37 & 168 & Hon. & $1300-1350$ \\
\hline S. maculatus & $M:(n=4) ; H:(n=2)$ & $29-37 ; 23$ & $144 ; 44$ & Nic. & $300-350$ \\
\hline M. norrisi & $M:(n=4) ; H:(n=3)$ & $82-92 ; 84-87$ & $7850 ; 5092$ & Hon.; Pan. & $100-150$ \\
\hline S. dumeril & $M:(n=3) ; H:(n=3)$ & $32-74 ; 48-81$ & $4268 ; 8316$ & Pan.; Nic. & $100-300$ \\
\hline H. perlo & $M:(n=2) ; H:(n=1)$ & $75-82 ; 67$ & $2828 ; 1056$ & Pan. & $250-500$ \\
\hline
\end{tabular}


Cuadro 3. Índice de equidad de Pielou e índice de Shannon-Wiener para las especies de condrictios capturados

Table 3. Pielou equity index and Shannon-Wiener diversity index of chondrichthyan species collected

\begin{tabular}{ccc}
\hline País & Pielou & Shannon \\
\hline Panamá & 0.623 & 1.644 \\
Costa Rica & 0.811 & 0.562 \\
Nicaragua & 0.891 & 2.051 \\
Honduras & 0.680 & 1.927 \\
Belice & 0.864 & 1.390 \\
\hline
\end{tabular}

Cuadro 4. Coeficiente de similitud de Jaccard (J) de especies de condrictios en el Caribe centroamericano

Table 4. Jaccard similarity coefficient (J) of species, in the Caribbean waters of Central America

\begin{tabular}{ccccc}
\hline País & Costa Rica & Nicaragua & Honduras & Belice \\
\hline Panamá & 0.14 & 0.20 & 0.35 & 0.27 \\
Costa Rica & - & 0.00 & 0.12 & 0.17 \\
Nicaragua & - & - & 0.23 & 0.15 \\
Honduras & - & - & - & 0.22 \\
\hline
\end{tabular}
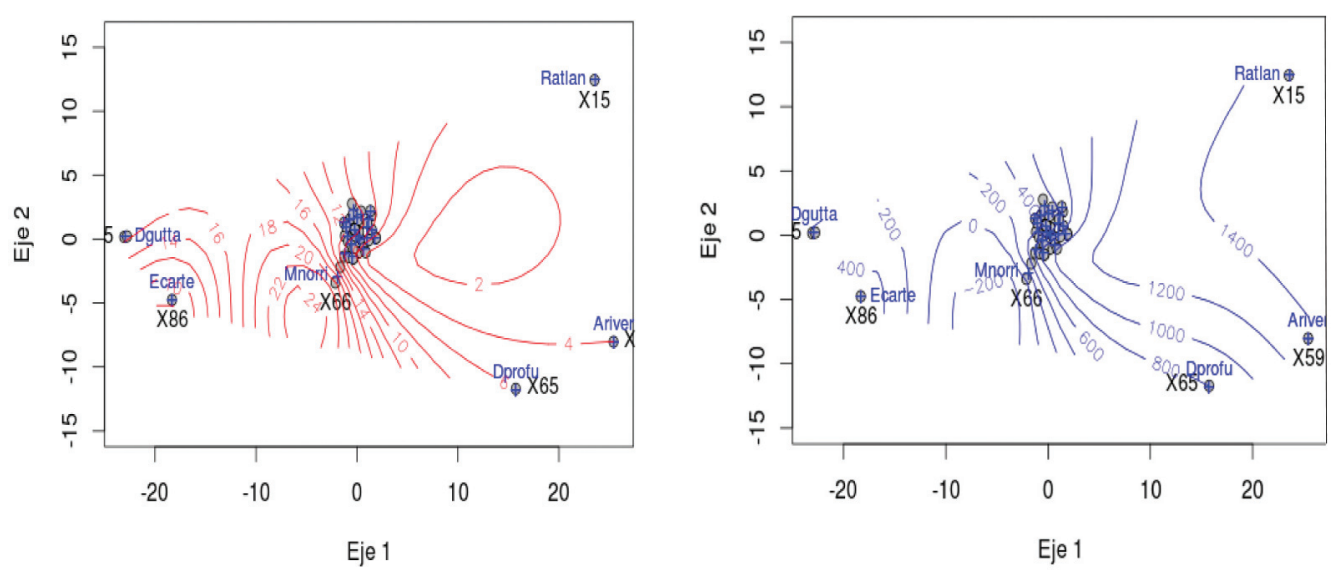

Fig. 6. Escalamiento multidimensional no métrico (NMDS) para todas las especies de condrictios capturados. Las especies que no se incluyen dentro de la agrupación central son: Dasyatis guttata, Etmopterus carteri, Mustelus norrisi, Deania profundorum, Apristurus riveri y Rhinochimaera atlantica. Contornos rojos (temperatura, ${ }^{\circ} \mathrm{C}$ ) y contornos azules (profundidad, $\mathrm{m}$ )

Fig. 6. Non-metric multidimensional scaling (NMDS) for all chondrichthyan species collected. Species not included in the main group are: Dasyatis guttata, Etmopterus carteri, Mustelus norrisi, Deania profundorum, Apristurus riveri and Rhinochimaera atlantica. Red contours (temperature, ${ }^{\circ} \mathrm{C}$ ) and blue contours (depth, m) 

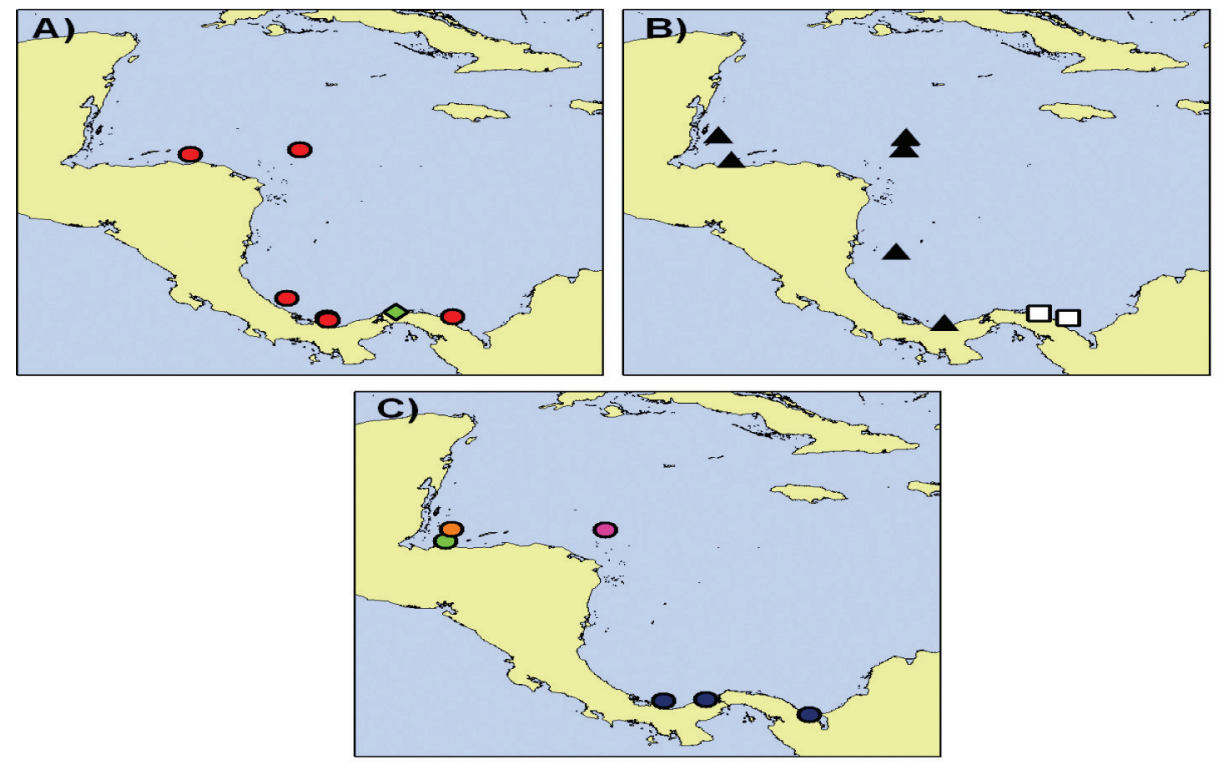

Fig. 7. Distribución espacial de los nuevos registros de condrictios en el Caribe centroamericano. (A) H. mirabilis $(\bullet)$ y R. atlantica $(\diamond)$. (B) E. gracilispinis $(\mathbf{\Delta})$ y $C$.

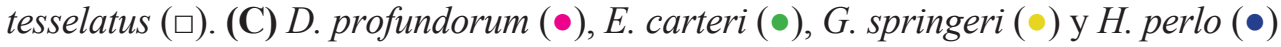

Fig. 7. Spatial distribution of new chondrichthyes recorded along the Caribbean coasts of Central America. (A) H. mirabilis $(\bullet)$ and R. atlantica $(\diamond)$. (B) E. gracilispinis $(\mathbf{\Delta})$ and $C$. tesselatus $(\square)$. (C) D. profundorum $(\bullet)$, E. carteri $(\bullet)$, G. springeri $(\bullet)$ and H. perlo $(\bullet)$

Es de aguas profundas y habita entre los 500 y 1500 m. Se encuentra distribuida en todo el Atlántico, pero sus aislados reportes se centran en datos de capturas incidentales en actividades pesqueras en costas del Golfo de México, Canadá y la costa este del Atlántico. Se capturó solo una hembra en Panamá (LT: $79 \mathrm{~cm}$ ). Se estima que puede ser abundante en profundidades no alcanzables para la flota pesquera de aguas profundas (UICN, 2011b).

Deania profundorum (Smith \& Radcliffe, 1912)

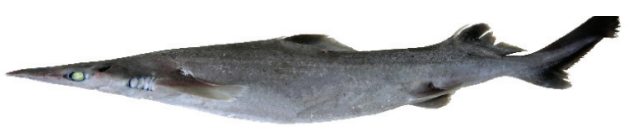

Presenta una distribución geográfica amplia, la cual la ubica en ambos lados del Atlántico, desde el oeste del Sahara hasta Suráfrica y desde Carolina del Norte hasta el Golfo de México, así como también en las Antillas (Compagno et al. 1989), Cabo Verde (Reinier, 1996), Azores (Santos et al. 1997), las Islas Canarias (Brito et al. 2002) y Portugal (Coelho \& Erzini, 2006). También ha sido reportada en el oeste de la India y en Filipinas (Compagno, 1998). En el presente estudio se capturó un ejemplar frente a las costas de Honduras (LT: $70 \mathrm{~cm}$ ), entre los 750 y $800 \mathrm{~m}$. Por su tamaño no es de interés comercial, y 
se le ha documentado en descartes de pesquerías de arrastre en varios lugares del mundo (UICN, 2011c).

\section{Centrophorus tesselatus}

Garman, 1906

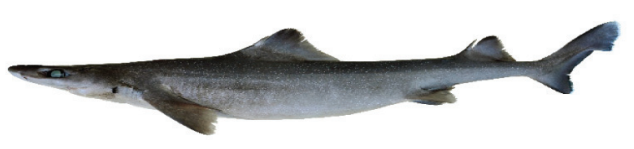

$\mathrm{Su}$ distribución lo ubica en el Pacífico central, en las Islas de Hawai y en el Pacífico noroeste: sureste de Honshu, Japón (Compagno, 1984a). Para el Atlántico se ha reportado únicamente en las costas de México y Colombia (Herndon et al. 2006). Todos los ejemplares se capturaron en Panamá, a los $900 \mathrm{~m}$, ampliando el rango batimétrico de la especie. Es pequeño, sin aleta anal, con dos aletas dorsales armadas por una fuerte espina cada una. Según Compagno (1984a), su talla máxima alcanza los $89 \mathrm{~cm}$, sin embargo, en el presente estudio se capturó una hembra de $96 \mathrm{~cm}$. Habita aguas entre los $20 \mathrm{~m}$ y $730 \mathrm{~m}$ (Kyne \& Simpfendorfer, 2007). A diferencia de los demás miembros del género Centrophorus se conoce poco sobre la biología de esta especie y tiene poca importancia en las pesquerías.

Etmopterus gracilispinis Krefft, 1968

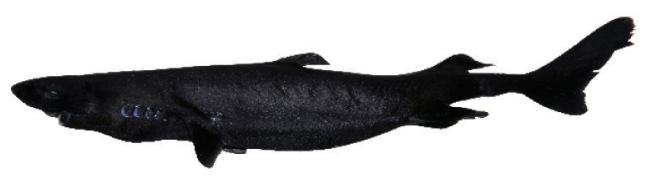

El género Etmopterus es el más diverso de la familia Squalidae, con al menos 31 especies descritas
(Compagno et al. 2005). Las especies de este género se conocen comúnmente como "gatas" o "tiburones linterna", se encuentran ampliamente distribuidos en aguas profundas de todos los océanos. Generalmente, son asociados a taludes insulares o continentales, entre los 70 y $1000 \mathrm{~m}$ y donde suelen concentrarse alrededor de los $800 \mathrm{~m}$ de profundidad (Yano \& Tanaka, 1989). También se le considera un tiburón pelágico y mesopelágico. Se distribuye geográficamente en el Atlántico noroeste (Virginia, Florida), así como en Uruguay y Argentina. También en el Atlántico este (Suráfrica). En el presente estudio se encontraron tres especies de este género. Para E. gracilispinis se capturaron 17 ejemplares: 6 machos (26-53 cm LT) y 11 hembras (26-106 cm LT) en todos los países, a excepción de Costa Rica, entre los 750 y 1150 $\mathrm{m}$, ampliando el ámbito de longitud total del género, así como su rango batimétrico. Su coloración es café oscuro por encima, con el reverso del hocico y el abdomen negros.

Etmopterus carteri Springer \& Burgess, 1985

Especie descubierta hace 30 años

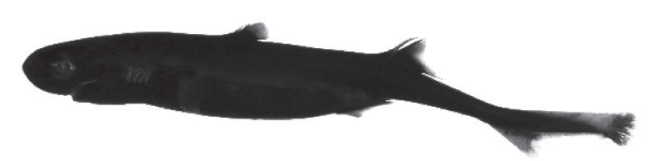

y poco conocida. Tiburón pequeño de aguas profundas que habita la plataforma continental a profundidades entre los 283 y 356 m (Springer \& Burgess, 1985). El ejemplar único del presente estudio se capturó entre 
los 400 y $500 \mathrm{~m}$ de profundidad, ampliando el ámbito batimétrico de la especie. La talla máxima total alcanza los $21.2 \mathrm{~cm}$ para los machos, y los 19 $\mathrm{cm}$ para las hembras (Compagno et al. 2005), talla superada por los $21 \mathrm{~cm}$ que alcanzó la hembra capturada en este muestreo. E. carteri es un tiburón de color negro intenso, posee unos ojos grandes de color verde, dos aletas dorsales a las que preceden dos fuertes espinas y un hocico redondeado.

Galeus springeri Konstantinou \& Cozzi, 1998

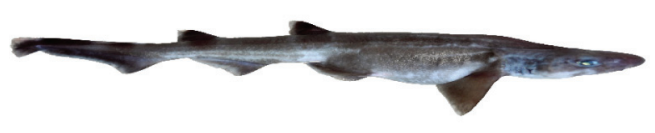

Tiburones relativamente pequeños, los machos pueden alcanzar los 31.8 cm LT y las hembras los $43.8 \mathrm{~cm}$ LT. Se capturó una hembra en Belice (52 cm LT), con lo cual se amplía el ámbito de la longitud total de la especie. El hábitat de esta especie se ha caracterizado por estar entre los 450 y 700 $\mathrm{m}$ de profundidad, lo cual coincide con los 500 y $550 \mathrm{~m}$ a los que se encontró en el presente estudio. Su distribución geográfica se limita a las Pequeñas Antillas, Puerto Rico, la costa norte de la Española (Haití y República Dominicana) y Cuba (Heupel, 2009).

Heptranchias perlo (Bonaterre, 1788)

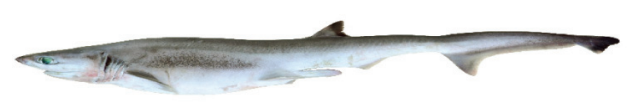

Cuerpo relativamente pequeño, con ojos grandes, cabeza angosta, siete branquias y con una sola aleta dorsal. Su talla máxima es 137 cm LT, para los machos, y $140 \mathrm{~cm}$ LT para las hembras. Es una especie cosmopolita, excepto en el Pacífico nororiental. Los individuos capturados en el Caribe panameño fueron una hembra, de 67 cm LT, y dos machos, de $75 \mathrm{~cm}$ y 82 $\mathrm{cm}$ LT, respectivamente. Su hábitat es demersal y/o semipelágico y se han encontrado ejemplares entre los 0 y $1000 \mathrm{~m}$ de profundidad. Los especímenes capturados en el presente estudio se encontraron entre los $250 \mathrm{y}$ $300 \mathrm{~m}$. Se localiza principalmente en el exterior de la plataforma continental y en el talud continental.

En el Cuadro 5 se presentan las profundidades a que fueron capturadas las especies de condrictios no reportadas con anterioridad en el Caribe centroamericano, los rangos en los que se ubicaron las tallas de las especies y los valores reportados con anterioridad en la literatura, con el fin de presentar comparativamente los resultados obtenidos en el presente estudio.

\section{DISCUSIÓN}

Enelpresenteestudio se encontraron 28 especies pertenecientes a 5 familias, conformadas tanto por ejemplares de fondo como pelágicos. Esa diversidad de especies refleja la gran variedad de ambientes que conforman esta región (Miloslavich et al. 2010).

Las familias registradas en bases de datos en línea para esta región (Froese \& Pauly, 2014; UICN, 2011a) se confirman en este estudio, y se reporta por primera vez la presencia de un ejemplar 
perteneciente a la familia Hexanchidae en el Caribe centroamericano.

Algunas de las especies capturadas durante el muestreo han sido registradas previamente en las cercanías de las costas de Cuba, Golfo de México y algunos archipiélagos de Suramérica. Cabe destacar que directamente en el área muestreada se documenta la presencia de C. cubana en el Archipiélago de San Andrés. Esta especie es la única de siete reconocidas que conforman la familia Chimaeridae presente en el Atlántico occidental (Caldas et al. 2009b). Adicionalmente, la distribución de Schroderichthys maculatus había sido reportada solo para las costas caribeñas de Honduras y Nicaragua (Compagno, 1984b). Ambos reportes concuerdan con los resultados del presente estudio. Asimismo, esta investigación aporta ocho especies como primeros registros para el área, ampliando así el conocimiento de la comunidad íctica.

Cuadro 5. Rango de profundidad y talla para las ocho especies no reportadas con anterioridad en el Caribe centroamericano

Table 5. Depth and size range for the eight species not previously reported in the Caribbean waters of Central America

\begin{tabular}{lcccc}
\hline Especie & $\begin{array}{c}\text { Rango de tallas } \\
\text { por especie y sexo } \\
\text { capturadas (cm) }\end{array}$ & $\begin{array}{c}\text { Tallas máximas } \\
\text { por especie y } \\
\text { sexo reportadas } \\
\text { previamente (cm) }\end{array}$ & $\begin{array}{c}\text { Rango de } \\
\text { profundidad de } \\
\text { capturas (m) }\end{array}$ & $\begin{array}{c}\text { Rango de } \\
\text { profundidad } \\
\text { reportado } \\
\text { previamente } \\
\text { (m) }\end{array}$ \\
\hline H. mirabilis & H: $16-57 ;$ M: $36-57.5$ & NR: 80 & $500-1250$ & $450-1936$ \\
R. atlantica & H: 79 & H: $140 ;$ M: 112.7 & $1450-1500$ & $500-1500$ \\
$\begin{array}{l}\text { D. profundorum } \\
\text { C. tesselatus }\end{array}$ & I: 70 & H: $80 ;$ M: 67 & $750-800$ & $275-1785$ \\
E. gracilispinis & H: $26-106 ;$ : $: 26-53$ & H: $30 ; 3 ;$ M: 26 & $750-1150$ & $70-1000$ \\
E. carteri & H: 21 & H: $19 ;$ M: 21.2 & $450-500$ & $283-356$ \\
$\begin{array}{l}\text { G. springeri } \\
\text { H. perlo }\end{array}$ & H: 52 & H: $43.8 ;$ M: 31.8 & $500-550$ & $450-700$ \\
\hline
\end{tabular}

H: hembra, M: macho, NR: sexo no reportado.

Como se puede apreciar en la figura 1, el diseño del muestreo fue homogéneo, sin embargo, la longitud de la línea de costa y la batimetría del talud de cada país determinaron el número de lances en cada transecto. Debido a lo anterior, los valores de la abundancia fueron estandarizados con respecto al área arrastrada, resultando Panamá el país con el valor más alto (Fig. 3).
Además, por esa misma razón, los resultados en la riqueza fueron mayores en Honduras, con 17 especies (Fig. 4). Se destaca, además, la presencia de condrictios de profundidad a lo largo de toda el área muestreada.

El análisis de escalonamiento multidimensional no métrico sugiere que la mayoría de las especies capturadas en el Caribe centroamericano 
exhiben un comportamiento similar en cuanto a su distribución en la columna de agua. Se diferencia claramente la formación de un grupo de condrictios asociados que comparten características de temperatura y profundidad: Anacanthobatis americanus, Dasyatis americana, Breviraja nigriventralis, Gurgesiella atlantica, Dipturus bullisi, Cruriraja rugosa, Leucoraja yucatanensis, Hydrolagus mirabilis, $H$. alberti, C. cubana, N. carri, $S$. cubensis, Centrophorus granulosus, $C$. tesselatus, E. gracilispinis, E. schultzi, Galeus springeri, G. arae, Apristurus parvipinnis, $S$. maculatus, $H$. perlo y Squatina dumeril. Asimismo, fuera de este agrupamiento se distinguen al menos 6 especies que se salen del rango que caracteriza a las capturas de condrictios en la zona de estudio: Dasyatis guttata, E. carteri, M. norrisi, D. profundorum, $A$. riveri y $R$. atlantica.

La riqueza y biomasa de los condrictios del talud continental de Chile se encuentran dominadas por los tiburones, especialmente del orden Squaliformes (Andrade \& Pequeño, 2008), lo que concuerda con lo obtenido en el presente estudio. Sin embargo, cuando se compara con el ensamblaje de condrictios del Caribe colombiano, se tiene que Acevedo et al. (2007) describen una mayor dominancia del orden Rajiformes.

La mayoría de las especies del muestreo corresponden a tiburones de profundidad, pero también se capturaron especies pelágicas y de aguas someras. Las especies pelágicas como S. cubensis y C. granulosus son de importancia comercial, se distribuyen en todo el Caribe, realizan migraciones y son especies conocidas. Lavulnerabilidad de todas esas especies de tiburones se debe principalmente a la sobrepesca y pesca incidental, así como a sus características biológicas: bajo potencial reproductivo y crecimiento lento, lo cual son factores que se deben tener en cuenta para los planes de manejo, conservación y protección de las especies.

En el Atlántico noroccidental figuran datos importantes sobre las disminuciones de las poblaciones de tiburones tanto costeros como oceánicos (Baum et al. 2003). Según esa revisión, una de las áreas accesadas por la flota pesquera de línea larga de los Estados Unidos corresponde al Caribe. Sin embargo, se calculó que entre los años 1986 y 2000 las faenas de pesca en esa área estuvieron restringidas a la región centro-oriental y no superaron las 100, lo cual representa una cifra relativamente pequeña, si se compara, por ejemplo, con el Golfo de México (más de 800 faenas) para el mismo período. En la actualidad, el efecto de la explotación sobre los tiburones de profundidad en muchas poblaciones del Caribe centroamericano se mantiene incierto.

El manejo y la conservación de los condrictios, en general, se han visto obstaculizados por la falta del conocimiento de su estado o incluso por la dirección de la tendencia de la población (Baum et al. 2003). La falta de información sobre estas especies está asociada al elevado costo de los 
monitoreos de sus poblaciones, y a que son especies altamente migratorias.

Cerca del $50 \%$ de las capturas globales estimadas de condrictios se deben a la pesca incidental, datos que no aparecen registrados en las estadísticas de pesca y que, además, son totalmente inmanejables (Stevens et al. 2000). Para el caso específico de los tiburones, un estudio del 2005 (Hareide et al. 2007) reveló que la pesquería dedicada a tiburones de profundidad del Atlántico norte presentó una disminución del $30 \%$, y estaba centrada sobre todo en las especies $C$. squamosus y C. granulosus, las cuales inicialmente se comercializaban frescas. Las pesquerías de profundidad se iniciaron en la década de los sesenta, cuando se empezó a observar un declive en la producción de las pesquerías tradicionales sobre el talud continental. A pesar de la poca información sobre la biología de esas especies, existen varios artículos publicados sobre algunos parámetros de reproducción que están citados en Paiva et al. 2011.

En el reciente trabajo de Kyne et al. (2012), relacionado con el estado de conservación de condrictios en América del Norte, América Central y el Caribe, los autores sugieren que los gobiernos de dichos países están urgidos, entre otros aspectos, de la recolección de información sobre datos de pesca, incluidos los descartes, así como impulsar estudios que amplíen el conocimiento en cuanto a las especies que habitan sus aguas. En este sentido, los resultados de la presente investigación contribuyen a solventar esa urgencia.
Además, el hallazgodelosprimeros registros amplía notablemente el rango de distribución de las especies, así como el conocimiento de la biodiversidad íctica marina del Caribe centroamericano, sin embargo, se deja consignado que es necesario realizar un mayor número de estudios en aguas del Caribe, para contribuir al conocimiento científico de la región.

\section{AGRADECIMIENTOS}

Este trabajo se realizó bajo los auspicios de la Universidad Nacional a través del Programa SERIO (Servicio Regional de Información Oceanográfica). A Mario González Recinos, Director de OSPESCA por permitir el uso de la base de datos. A la tripulación y al grupo de investigadores del B/O Miguel Oliver, de la Secretaría del Mar de España. A Jorge Cortés del CIMAR por los aportes realizados a la mejora del presente documento.

\section{BIBLIOGRAFÍA}

Acevedo, K., Bohórquez-Herrera, J., Moreno, F., Moreno, C., Molina, E., Grijalba-Bendeck, M. \& Gómez-Canchong, P. (2007). Tiburones y rayas (Subclase Elasmobranchii) descartados por la flota de arrastre camaronero en el Caribe de Colombia. Acta Biol. Colombia, 12(2), 69-80.

Alvarado, J. J., Solís-Marín, F. A. \& Ahearn, C. ${ }^{\dagger}$ (2008). Equinodermos (Echinodermata) del Caribe Centroamericano. Rev. Biol. Trop., 56(Suppl. 3), 37-55.

Andrade, I. \& Pequeño, G. (2008). Mesobathic chondrichthyes of the Juan Fernández seamounts: are they different from 
those of the central Chilean continental slope? Rev. Biol. Trop., 56(1), 181-190.

Bascompte, J., Melián, C. J. \& Sala, E. (2005). Interaction strength combinations and the overfishing of a marine food web. P. Natl. Acad. Sci. USA, 102(15), 5443-5447. DOI: http:// dx.doi.org/10.1073/pnas.0501562102

Baum, J. K., Myers, R. A. M., Kehler, D. G., Worm, B., Harley, S. J. \& Doherty, P. A. (2003). Collapse and Conservation of Shark Populations in the Northwest Atlantic. Science, 299, 389392. DOI: http://dx.doi.org/10.1126/ science. 1079777

Borcard, D., Gillet, F. \& Legendre, P. (2011). Spatial analisys of ecological data. En D. Bocard, F. Gillet \& P. Legendre (Eds.), Numerical Ecology with $R$ (pp. 227292). New York, EE.UU.: Springer.

Brito, A., Pascual, P. J., Falcón, J. M., Sancho, A. \& González, G. (2002). Peces de las Islas Canarias. Catálogo comentado e ilustrado. Tenerife, España: Francisco Lemus.

Caldas, J. P., Díaz-Trujillo, E. M., García, C. B. \& Duarte, L. O. (2009a). Revisión Histórica de la Pesca de Tiburones y Rayas en el Mar Caribe Continental de Colombia. En V. A. Puentes, F. Navia, P. A. Mejía-Falla, J. P. Caldas, M. C. DíazGranados \& L. A. Zapata (Eds.), Avances en el conocimiento de tiburones, rayas y quimeras de Colombia (pp. 97131). Santa Marta, Colombia: Ministerio de Ambiente, Vivienda y Desarrollo.

Caldas, J. P., Didier, D. A., Santos-Martínez, A. \& Acero, A. P. (2009b). New record of Chimaera cubana HowellRivero (Chondrichthyes: Holocephali: Chimaeridae) from the western Caribbean Sea. Pan-Am Jour Aq Sci., 4(4), 583-588.
Coelho, R. \& Erzini, K. (2006). On the occurrence of the arrowhead dogfish Deania profundorum (Chondrichtyes: Squalidae) off southern Portugal, with a missing gill slit. Cybium, 30(1), 93-96.

Compagno, L. J. V. (1984a). FAO species catalogue. Vol. 4. Sharks of the World. Part 1. Hexanchiformes to Lamniformes. Rome, Italy: FAO Fish Synop.

Compagno, L. J. V. (1984b). FAO species catalogue. Vol. 4. Sharks of the World. Part 2. Carcharhiniformes. Rome, Italy: FAO Fish Synop.

Compagno, L. J. V., Ebert, D. A. \& Smale, M. J. (1989). Guide to the Sharks and Rays of southern Africa. London, England: Illustrated.

Compagno, L. J. V. (1998). Sharks. En K. E. Carpentier \& V. H. Niem (Eds.), The living marine resources of the Western Central Pacific. Vol. 2: Cephalopods, crustaceans, holothurians and Sharks (pp. 1193-1366). Rome, Italy: FAO.

Compagno, L. J. V., Dando, M. \& Fowler, S. L. (2005). Sharks of the World. London, England: Collins Press.

Ebert, D. A. \& Compagno, L. J. V. (2007). Biodiversity and systematics of skates (Chondrichthyes: Rajiformes: Rajoidei). Environ. Biol. Fish., 80(2-3), 111124. DOI: http://dx.doi.org/10.1007/ s10641-007-9247-0

Froese,R.\&Pauly,D.(Eds.).(2014).FishBase. World Wide Web electronic publication. Recuperado en septiembre 19, 2011, disponible en http://www.fishbase.org/ González, C., Teruel, J., López, E. \& Paz, X. (2007, setiembre). Feeding Habits and Biological Features of Deep-Sea Species of the Northwest Atlantic: Large-eyed Rabbitfish (Hydrolagus mirabilis), Narrownose Chimaera 
(Harriotta raleighana) and Black Dogish (Centroscyllium fabricii). Ponencia presentada en Scientific Council Meeting. Centro Oceanográfico de Vigo, Galicia, España.

Hareide, N. R., Carlson, J., Clarke, M., Clarke, S., Ellis, J., Fordham, S., Fowler, S., Pinho, M., Raymakers, C., Serena, F., Seret, B. \& Polti, S. (2007). European Shark fisheries: a preliminary investigation into fisheries, conversion factors, trade products, markets and management measures. Bruselas, Bélgica: European Elasmobranch Association. DOI: http://dx.doi. org/10.1111/j.1444-2906.2007.01360.x

Hernández, H. H. \& Núñez, R. S. (1998). Nuevo registro para el Caribe Sur Colombiano del tiburón Quelvacho Centrophorus granulosus (Schneider) (Pisces: Centrophoridae). Bol. Invest. Mar. Cost., 27, 107-112.

Herndon, A. P., Horodysky, A. Z. \& Burgess, G. H. (2006). Etmopterus schul$t z i$. IUCN Red List of Threatened Species. Versión 2013.1. Recuperado en noviembre 20, 2011, disponible en http://iucnredlist.org.

Heupel, M. R. (2009). Galeus springeri. IUCN Red List of Threatened Species. Versión 2013.1. Recuperado en febrero 21, 2012, disponible en http://iucnredlist.org.

Kyne, P. M. \& Simpfendorfer, C. A. (2007). A collation and summarization of available data on deepwater chondrichthyans: biodiversity, life history and fisheries. Brisbane St. Lucia, Australia: Marine Conservation Biology Institute. Kyne, P. M., Carlson, J. K., Ebert, D. A., Fordham, S. V., Bizzarro, J. J., Graham, R. T., Kulka, D. W., Tewes, E. E., Harrison, L. R. \& Dulvy, N. K. (2012). The Conservation Status of North American, Central American, and
Caribbean Chondrichthyans. Vancouver, Canada: IUCN Species Survival Commission Shark Specialist Group.

Konstantinou, H., McEachran, J. D. \& Woolley, J. B. (2000). The systematics and reproductive biology of the $\mathrm{Ga}$ leus arae subspecific complex (Chondrichthyes: Scyliorhinidae). Environ. Biol. Fish., 57, 117-129. OI: http:// dx.doi.org/10.1023/A:1007600228078

Large, P. A., Hammer, C., Bergstad, O. A., Gorgon, J. D. M. \& Lorance, P. (2003). Deep water fisheries of the Northeast Atlantic: II Assessment and Management Approaches. J. Northw. Atl. Fish. Sci., 31, 151-163.

Magurran, A. E. (1988). Diversity indices and species abundance models. En A. Magurran (Ed.), Ecological diversity and its measurement (pp. 7-47). Cambridge, England: University Press.

Mejía-Falla, P. A., Navia, A. F., MejíaLadino, L. M., Acero, A. P. \& Rubio, E. A. (2007). Tiburones y rayas de Colombia (Pisces Elasmobranchii): Lista actualizada, revisada y comentada. Bol. Invest. Mar. Cost., 36, 111-149.

Miloslavich, P. \& Klein, E. (2005). Linking marine biodiversity research and Conservation in the Caribbean. En P. Miloslavich \& E. Klein (Eds.), Caribbean Marine Biodiversity: The Known and the Unknow (pp. 1-25). Pennsylvania, EE.UU.: DEStech, Lancaster.

Miloslavich, P., Díaz, J. M., Klein, E., Alvarado, J. J., Díaz, C., Gobin, J. \& Ortiz, M. (2010). Marine Biodiversity in the Caribbean: Regional Estimates and Distribution Patterns. Plos One. 5(8), e11916. doi:10.1371/journal.pone.0011916

Moreno, C. E. (2001). Métodos para medir la biodiversidad. En M\&T-Manuales y Tesis SEA, Vol. 1. Zaragoza, España: GORFI, S. A. 
Nelson, J. S. (2006). Class Chondrichties. En J. Nelson (Ed.), Fishes of the world (pp. 39-82). 4th edition. New Jersey, EE.UU.: Wiley \& Sons, Inc. DOI: http://dx.doi.org/10.1017/ S0009838806000309

Reinier, F. (1996). Catálogo dos peixes de Archipiélago do Cabo Verde. Vol. 2. Lisboa, Portugal: Publicaçõcoes avulsas do IPIMAR.

OSPESCA, Organización del sector pesquero y acuícola de Centroamérica. (2009). Propuesta de investigaciones pesqueras de aguas profundas en ambas costas de Centroamérica. San Salvador, El Salvador: OSPESCA.

Paiva, R. B., Neves, A., Sequeira, V. \& Gordo, L. S. (2011). Reproductive parameters of the commercially exploited deep-water shark, Deania calcea (Centrophoridae). Cybium, 35(2), 131-140.

Peet, R. K. (1975). Relative diversity indices. Ecology, 56(2), 496-498. DOI: http://dx.doi.org/10.2307/1934984

Pielou, E. C. (1975). Ecological Diversity. New York, EE.UU.: John Wiley \& Sons. Santos, R. S., Porteiro, F. M. \& Barreiros, J. P. (1997). Marine Fishes of the Azores: An annotated checklist and bibliography. Azores, Portugal: Nova Gráfica.

Springer, S. \& Burgess, G. H. (1985). Two new Dwarf Dogsharks
(Etmopterus, Squalidae), Found off the Caribbean Coast of Colombia. Copeia, 3, 584-591. DOI: http://dx.doi. org $/ 10.2307 / 1444748$

Stevens, J. D., Bonfil, R., Dulvy, N. K. \& Walker, P. A. (2000). The effects of fishing on sharks, rays, and chimaeras (chondrichthyans), and the implications for marine ecosystems. J. Mar. Sci., 57(13), 476-494.

Tavares, R. (2009). Análisis de la abundancia, distribución y tallas de tiburones capturados por pesca artesanal en el parque Nacional Archipiélago Los Roques, Venezuela. Interciencia, 34(7), 463-470.

UICN. (2011a). Red List of Threatened Species. Recuperado en septiembre 19, 2011, disponible en http://www. iucnredlist.org/

UICN. (2011b). Red List of Threatened Species. Recuperado en septiembre 19, 2011, disponible en http://www. iucnredlist.org/details/60145/0

UICN. (2011c). Red List of Threatened Species. Recuperado en septiembre 19, 2011, disponible en http://www. iucnredlist.org/details/161551/0

Yano, K. \& Tanaka, S. (1989). Hermaphroditism in the lantern shark, Etmopterus unicolor (Squalidae, Chondrichthyes). Jpn. J. Ichthyol., 36(3), 338-345. 